Number of pages: 14

Number of tables: 1

Number of figures: 2

Number of references: 3

\title{
Predicting and controlling risks from human exposures to vibration and mechanical shock: flag waving and flag weaving
}

Michael J Griffin

Human Factors Research Unit

Institute of Sound and Vibration Research

University of Southampton

Southampton SO17 1BJ

United Kingdom

Telephone: +44 (0)23 80592277

e-mail: M.J.Griffin@soton.ac.uk 


\begin{abstract}
At work or in leisure activities, many people are exposed to vibration or mechanical shocks associated with risks of injury or disease. This paper identifies information that can be used to decide whether there may be a risk from exposure to hand-transmitted vibration or whole-body vibration and shock, and suggests actions that can control the risks. The complex and time-varying nature of human exposures to vibration and shock, the complexity of the different disorders, and uncertainty as to the mechanisms of injury and the factors influencing injury have prevented the definition of doseresponse relationships well-proven by scientific study. It is necessary to wave a flag indicating when there is a need to control risks from exposure to vibration and shock while scientific enquiry provides understanding needed to weave a better flag. It is concluded that quantifying exposure severity is often neither necessary nor sufficient to either identify risks or implement measures that control the risks.
\end{abstract}

\title{
Practitioner summary
}

The identification of risks associated with exposure to vibration and mechanical shock cannot, and need not, rely solely on the quantification of exposure severity. Qualitative methods can provide a sufficient indication of the need for control measures, which should not be restricted to reducing standardised measures of exposure severity.

\section{Keywords:}

Whole-body vibration; hand-transmitted vibration; mechanical shocks; health and safety; risks; control measures. 


\section{Introduction}

The motions associated with human exposures to vibration and mechanical shocks are often complex and variable. Hand-transmitted and whole-body vibration and shock can damage the body but the types of damage are also complex and variable, and poorly understood. It seems that many people may be at risk of disorder from exposures to vibration and shock at work or during leisure activities. It is therefore desirable to control the risk that any of the various disorders may appear or progress.

Risk is the probability that a person will experience an adverse health effect if exposed to a hazard (e.g., vibration or mechanical shock). When considering the risks associated with an exposure to vibration or shock it is natural to ask what adverse health effect might arise and what is the probability that it will occur. The role of science is to pose questions that can be answered, but with current understanding it is not possible to state with confidence the probability of any adverse effect arising from any exposure to vibration or shock. Nevertheless, the question begs an answer when making decisions, including deciding whether there is a significant risk or how to control an anticipated risk. There is therefore a tension between the honesty of 'don't know' and the desire to be helpful with a 'best guess'.

If a disorder arises from cumulative exposure (rather than a discrete event) it may be assumed to be caused by a dose of vibration and shock. Unlike an average measure of exposure, which can increase or decrease over time, a measure of dose can only increase, unless there is an allowance for recovery during periods of low exposure. Various components in the motion (e.g., different magnitudes, frequencies, directions, and durations) will contribute to the dose, which might be measured or estimated over different durations (e.g., a working day or a lifetime).

The scientific approach is to seek a relation between a measure of dose and a measure of an identified disorder. In practice this involves selecting a measure of the disorder (e.g., the probability of a specified severity of the specified disorder) and seeking the measure of dose that is most closely associated with the measure of disorder. This requires epidemiological or experimental research or modelling of the mechanisms involved, or some combination of these alternatives. With the complexities involved in human responses to vibration and shock this is not a short journey.

The underlying tension is between pretence that it is known how to predict and control risks and the contrary view that few of the risks are understood and so methods of identifying and controlling risks are limited in their precision. With the uncertainty in 
mind, this paper attempts to identify what information can be used to predict risks and what methods can be used to control risks.

\section{The nature of risks and their cause}

\subsection{Understanding the nature of disorders}

A pre-requisite for quantifying or controlling risks is an understanding of the disorder caused by vibration and shock. Unfortunately, the nature and pathogenesis of the problems associated with exposures to vibration and mechanical shock are poorly understood. Exposures to hand-transmitted vibration result in a collection of disorders called the hand-arm vibration syndrome (HAVS) comprising vascular, neurological, muscular, articular, and perhaps other problems (Griffin and Bovenzi 2002). In some countries vibration-induced white finger (VWF) is the most recognised disorder but there is insufficient understanding of VWF and the mechanisms resulting in VWF for the understanding to be used to provide confident predictions of how VWF depends on the magnitude, frequency, direction, and duration of motion, or other factors (Griffin et al. 2003). Exposures to whole-body vibration and mechanical shock are associated with back pain, but the phenomenon causing back pain, or even the location of any damage causing back pain, is not agreed, and dose response relationships are still being sought (e.g., Bovenzi 2010). Only if the mechanism of damage is assumed (i.e., there is a model of the mechanism that predicts how damage will depend on the magnitude, frequency, direction, and duration of motion, etc.) is it reasonable to claim that back pain can be predicted from the magnitude, frequency, direction, and duration of motion, and other factors. Furthermore, there may be damage without pain and also pain without damage.

It might be thought that if an epidemiological study finds an association between the occurrence of a disorder and a measure of dose the measure of dose can be used to predict the risk of the disorder. However, a cross-sectional epidemiological study cannot establish a causal relationship. Longitudinal epidemiological studies can reveal causal associations but they should not be assumed to apply in other situations where there are different magnitudes, frequencies, directions, or durations of motion, or other variables that can be expected to influence the probability or severity of disorder.

Improved understanding of the pathogenesis of motion-induced disorders would give confidence to the development of hypothetical mechanisms for how vibration and shock cause injury, and models of those mechanisms, which may then be 'calibrated' using evidence from experimental and epidemiological studies. Such models may be 
expected to predict how the probability, or severity, of a specified disorder depends on the magnitude, frequency, direction, and duration of motion, and other factors (e.g., sitting posture, hand posture, and grip force). When there is sufficient evidence underlying each part of this process it may be possible to make useful predictions of the extent of any risk associated with a chronic exposure to vibration or shock. The predictions, which should have uncertainty associated with their assumptions, should be supported by evidence from studies in which they have been 'calibrated', and can be tested by further study and inform decision making if proven useful.

Uncertainty in the disorders associated with vibration and shock inevitably leads to uncertainty in the prediction of the disorders and uncertainty over the best means of controlling disorders.

\subsection{Evaluating the severity of exposures to vibration and shock}

Exposures to vibration and shock may be measured (i.e., converted to a form that can be recorded), evaluated (i.e., expressed by one or a few numbers reflecting severity, such as a measure of 'dose'), and assessed (judged relative to criteria). Measurement, evaluation, and assessment do not control risks: they are only useful if they lead to a better decision than would otherwise have been possible.

One approach to controlling risks is to determine, or assume, dose-response relationships that show the risks from different doses of vibration. This should encourage study to determine useful methods of measuring and evaluating vibration and shock so that a reliable relation is determined between the 'evaluations' and the risk of a specific disorder. In practice, the problem is complex because of the large influence of several different variables that tend to co-vary within any population.

If associations between various doses of vibration and the risks of various disorders were well established, dose-response information could be used to set a threshold for the acceptable dose by selecting an acceptable risk. Suitable measurement, evaluation, and assessment of exposures could then help to: (i) identify risks, (ii) quantify risks, and (iii) indicate some methods for reducing risks. When the association between the dose of vibration and a disorder is not understood, or the disorder is not known, the measurement, evaluation, and assessment of exposures is of limited value. In some situations the measurement of exposures can detract from, and delay, actions that can be taken without measuring, evaluating, and assessing the vibration or shock. Furthermore, a single procedure for predicting risk is insufficient or misleading if, as is 
often the case, there can be more than one type of disorder caused by vibration or shock.

\subsection{Dose-response information}

A range of adverse consequences may arise from exposures to vibration and shock and it is likely that many factors influence the outcome from such exposures. With various outcomes, often ill-defined, the consequences of exposure can be difficult to quantify. In these circumstances, it would be optimistic to expect that dose-response information can be a sufficient or reliable indication of acceptable risk. Epidemiological studies investigating dose-response associations are essential to advance understanding but the application of the findings from one study to another situation requires assumptions and merits caution.

There are many reasons why measurements of vibration and shock currently provide only weak indications of risk. Understanding of the relation between motion and risk is limited, and risk depends on many factors additional to the motion (e.g., posture and individual susceptibility). Measurements may not 'capture' the motion presenting risk. Extreme shocks, rough surfaces, or heavy seas that are rarely encountered will be associated with the greatest risk but unlikely to be measured. Similarly, occasional extreme exposure to hand-transmitted vibration will be more likely to cause the handarm vibration syndrome than the 'average' exposure to hand-transmitted vibration that is more likely to be measured. Furthermore, motion evaluation methods will not always encourage appropriate control measures (by designers, engineers, and management) because they must be simple to allow convenient application and so exclude some factors expected to influence risk (e.g., posture, grip, nonlinearities in response).

The term 'validation' is often used when a finding conforms to a prediction, but if many variables influence the prediction there can be little confidence in the claimed association, and no confidence that the finding corroborates the influence of variables that have not been investigated. A statistically significant association between a measure of motion and the probability of disorders, or the severity of disorders, does not 'validate' the measure of motion. Increased motion will tend to increase the risk and increase many unreasonable measures of motion severity: causal associations are required, not merely statistically significant associations.

\subsection{Identification of risk}

If a standardised method of evaluating motion severity exists it might seem obvious that an indication should be provided showing when the risks of exposure become 
unacceptable. However, this ignores the quality of the evidence that influenced the motion evaluation method, which may be merely a consensus of opinion with unspecified evidence of an association with risk.

Recognising that there are many factors involved in a dose-response relationship (Figure 1), a logical process for developing standardised dose-response information is shown in Table 1. This may be a logical approach, and there may be no alternative, but it may be dismissed as impractical because it requires unachievable understanding of the associations between the risks of injury and exposure to vibration and shock. Nevertheless, the 'test of honesty' in the final step remains important: a dose-response relationship promulgated by scientists or standardisation bodies should seek to make it clear, and not conceal, what is 'known' and what is 'assumed' in the relationship.

\section{FIGURE 1 AND TABLE 1 ABOUT HERE}

Even if there was confidence in a dose-response relationship, the level at which the risk is considered unacceptable may be based on the economic or political consequences of setting a lower or higher value, not the actual risk to those exposed (whether or not it is known). Whereas the role of the scientist is to offer methods of measuring and evaluating motion it is the role of the politician to decide on 'limits'. Even limits based on quantifiable risk will be influenced by practicalities and not founded entirely in 'science'. It follows that any such limits may not be reliable indications of risk. Notwithstanding large uncertainties, imperfect evaluation methods might be used to limit exposures to below a precise value because such a limit 'raises a flag' indicating foreseeable risk and thereby heightens awareness. It is helpful if the measurement and evaluation associated with a limit is simple, although achieving a lower value should be expected to reduce risks. Arguably, an $A(8)$ based on r.m.s. acceleration fails this test when exposures to either whole-body vibration or hand-transmitted vibration includes short exposures to vibration or occasional shocks - r.m.s. averaging undoubtedly allows excessive exposure to such motions (Griffin, 2004).

Across a broad spectrum of exposures, the same daily exposure will not present the same risk in all situations. Should the same limit be used to indicate risk irrespective of age and fitness, years of exposure, the priority of the activity, and the ease of controlling exposure to vibration and shock in different scenarios? Clearly the same 'limit' will not be appropriate in all situations, but it is always appropriate to reduce the exposure to the 'lowest practical level'. 
Even with standardised methods of measuring, evaluating, and assessing exposures to motion, there remain large uncertainties when measuring exposures, large assumptions in the method of evaluating exposures, and considerable uncertainty in assessing risks. Research can advance understanding and eventually provide more accurate methods of predicting each of the many different problems that are associated with exposures to whole-body and hand-transmitted vibration and shock. However, it may be unreasonable and even unhelpful to standardise either complex methods or alternative methods of measuring, evaluating, and assessing exposures to vibration and shock. For example, a better method can probably be evolved to predict vibrationinduced white finger, but if this involves a frequency weighting whose application will allow greater vibration at some frequencies it may increase the incidence of other disorders associated with hand-transmitted vibration. It seems reasonable to suggest that minor refinements to any method of evaluation will not provide useful improvements to the prediction of risk if some factors known to influence risk by a greater amount are ignored. If large changes are made to methods of predicting risks it would seem appropriate to undertake a 'risk assessment' to consider the possibility that the changes may increase the risks.

With many uncertainties, for practical purposes it may be sufficient to seek a rough indication of risk - an indication sufficient to decide whether further action and investigation is warranted. Limitations to understanding are such that the 'identification of risk' cannot depend solely on the 'prediction of the extent of risk' from standardised methods of measurement, evaluation, and assessment.

\section{Identifying and controlling risks}

A pre-requisite for the control of risks is the identification that there may be a risk. The initial question to be answered during a risk assessment is whether there is exposure to whole-body or hand-transmitted vibration or mechanical shock. If there is a possibility of exposure, it becomes necessary to decide whether the risks are likely to be sufficient to require the implementation of control measures.

\subsection{Identifying possible risk}

To be practical, a 'risk assessment' cannot be dependent on an accurate understanding of the extent of any risk. The general nature of an environment may be sufficient to identify a potential risk - attempting to measure the motion may then delay, or distract from, appropriate action. As experience and understanding develop, associations between 'situations' (jobs, machines, tools, and environments) and risks 
become clearer, and some actions can be linked to 'situations' rather than measures of motion.

The question should not be 'does an exposure exceed a limit?' The question should be 'could the situation present a risk? If it is reasonably possible for vibration or mechanical shocks to present a significant risk it is appropriate to initiate some form of control measure - sometimes comprehensive measures but sometimes simple measures such as prohibiting operation in conditions likely to be associated with unnecessary risk.

It is possible to undertake comprehensive, time-consuming, and expensive measurements and evaluations of vibration and shock but fail to quantify the motion causing greatest risk. In part this is because the greatest risk arises from the most severe exposure, which occurs rarely. Measurements are generally made in 'typical' conditions, whereas greatest risk occurs in atypical conditions, such as when there are unusually high magnitudes of vibration, or rare but extreme shocks, or unusually long durations of exposure to vibration, or extreme postures, etc.

The 'raising of a flag' to signal the need for control measures becomes appropriate if it is known that a tool, machine, or form of transport is associated with risk. This knowledge might be informed by standardised measures of exposure severity, but it should also be influenced by health surveillance of various forms, by formal and informal reports of problems within the industry, and by scientific, engineering, and medical literature. The flag should be raised to warn of risk when such tools, machines, or forms of transport are used. If the flag is raised, control measures should seek to minimise risk by means that are appropriate to the situation.

Exceeding a 'limit' to daily exposure to motion may be sufficient to 'raise a warning flag', but no 'limit' can be a reliable indicator of risk. Control measures may be beneficial for a wide range of situations, not merely those for which sample measurements exceed a limit. There are other matters to consider, as suggested in Figure 2.

\section{FIGURE 2 ABOUT HERE}

It may be unwise, and unnecessary, to 'lower the flag' because vibration exposure is reduced below some limit. Limits are unreliable indicators of risk and control measures appropriate above a limit will often be appropriate below the limit. However, the balance between alternative control measures, and the method of implementing control measures, may change as a risk is reduced. 


\subsection{Controlling risks}

The control of risk first requires the identification of potential risk and then the identification of suitable control measures. If a possible risk is identified, it is appropriate to identify ways of preventing disorder, or at least preventing severe disorder.

When the flag is raised there should be effort to reduce the risks. A combination of measures such as those shown in Figure 2 can be expected to reduce the chances of developing a disorder induced by vibration or shock and, if any disorder develops, reduce the chances of the problem progressing.

If the nature of the disorder is well defined, and the causal factors are fully understood, the control of risks may include the manipulation of the relevant variables (e.g., magnitude of motion, duration of exposure, posture) to reduce risk, and health surveillance might be used to check that the risks have been adequately controlled. If the factors influencing the disorder are not well understood, any manipulation of variables has a more uncertain benefit. If the nature of the disorder is uncertain, health surveillance may be of limited value.

\section{Discussion}

By means of scientific study it is possible to build understanding and weave a picture of the nature of the risks and the factors influencing risks associated with exposure to vibration and mechanical shock. Meanwhile, standards present methods for quantifying the severity of exposures based on consensus. When seeking to identify and control the risks it may be necessary to consider matters outside the scope or the competence of the standards.

There can be a 'conflict' between 'science' that seeks to understand, and so identifies where there is lack of understanding, and the need for 'decisions' irrespective of the degree of understanding. A scientist who contributes to standardisation based on consensus is 'compromised', or has a conflict of interest between upholding the principal of collective responsibility while knowing that the outcome is at best an assumption and sometimes less than is possible with current understanding. The meeting of minds comes when the scientist is prepared to accept a 'best guess' because it is openly admitted to be only a best guess and not dressed up to be well proven.

During a risk assessment many hazards will be considered in addition to deciding whether there are risks associated with exposure to vibration and shock. The risks 
arising from occupational and leisure activities are often complex and specialised understanding cannot be expected among those performing risk assessments. With some risks, a quantitative assessment using standardised instrumentation may be sufficient to identify whether control measures are appropriate. With other risks, a qualitative assessment can be sufficient to identify whether there is a potential risk. Because exposures to vibration can be 'quantified' using standardised equipment it should not be assumed that 'qualitative' assessments are not also necessary.

\subsection{Quantitative methods of identifying risk and control measures}

Various standards 'unify methods' of quantifying the severity of human exposures to vibration and shock. Such standards may not convey the status of the evidence on which they are based, even between two extremes: (i) situations where there is good understanding and good agreement but a need to define the agreement, and (ii) situations where there is little understanding but still some advantage in presenting a consensus 'best guess'. In both cases the resulting standard can assist progress (e.g., allow the development of standardised methods and instrumentation for measuring and evaluating vibration and shock), but whereas the former can be relied upon, the latter cannot. There are large uncertainties associated with the appropriate frequency weightings, axis multiplying factors, and time-dependencies in standards for quantifying exposures to vibration and mechanical shock. Even if these were well known, the influence of intra-subject variability (e.g., posture) and inter-subject variability (e.g., susceptibility) are less well understood and there is uncertainty as to the relation between any chosen measure of the severity of motion and the risk of any type of harm.

If the uncertainties were expressed in the appropriate standards it would be clear that any limits or action levels associated with the standardised methods of evaluating vibration and shock give, at best, very approximate indications of risk: the body might be harmed by some exposures below an 'exposure action value' but might not be harmed by some exposures in excess of an 'exposure limit value'. The current failure to convey uncertainties associated with standardised methods of evaluating the severity of human exposures to vibration and shock is a deceit that will entertain future generations. Meanwhile, it would be wise to assume that any quantitative guidance on the extent of risk has a large degree of uncertainty. In practice this means that it is unwise to assume there is no risk, and therefore no need for action, merely because an exposure appears to be below an action value! 
Whereas standardised methods of 'evaluating' vibration and shock may support only very uncertain guidance on 'assessing' risks, an evaluation method can contain useful information for 'controlling' risks. An evaluation method should indicate some of the factors that can be changed to reduce risk: the relative merits of different magnitudes, frequencies, directions, and durations of motion. This will be used by those in control of the motion (e.g., designers of seats and suspensions) and those controlling exposures to motion (e.g., management). In practice this requires a transparent, reasonable, and continuous relation between these variables.

The weightings given to the characteristics of motion and other factors (i.e., the evaluation method) have can have more influence when 'controlling' risks than when 'assessing' risks. When deciding on weightings it therefore seems desirable to prioritise consideration of their influence on the 'control' of risks rather than their influence on the 'assessment' of risks. Whereas it is difficult to determine the absolute importance of motion (required for assessment) it is often easier to determine the relative importance of two motions (as required to define the weightings in a method of evaluation). The absolute significance is uncertain but the relative importance is better understood, and more useful when controlling the severity of vibration and shock.

If a method of evaluating motion places inappropriate 'weight' on some frequencies, directions, or durations of motion it will encourage inappropriate control measures, and may increase risks. In the context of current standards, this may apply to some applications of root-mean-square measures, some frequency weightings, some axis multiplying factors, and some restrictions to one or a few axes of motion. These limitations can fail to encourage optimum design. The exclusion of any factor from the motion assessment discourages the control of that factor to reduce risk (e.g., the optimisation of sitting posture).

\subsection{Qualitative methods of identifying risk and control measures}

If it is accepted that there is large uncertainty in methods of evaluating the severity of exposures to vibration and shock, and that methods of assessment based on these evaluations also have large uncertainty, measuring, evaluating and assessing motion exposures have limited value, and can be unnecessary. If a risk can be expected without quantifying the severity of the motion, and measures to control the risk do not depend on knowing the severity, it can be an expensive 'luxury' to try to quantify exposure severity. 
Some knowledge of vibration severity, as calculated from information declared by the supplier of a tool or machine, or available elsewhere, may inform a risk assessment and the consequent flag waving signifying that control measures are required, but the actual risk will also depend on other factors. For example, the risk may seem to be low, but only if there are control measures to prevent excessive exposure (e.g., unusually long durations of exposure or operation in unusual conditions).

It might be helpful to know the severity of the motion so as to assist a decision between alternatives (e.g., which machine, which tool, or which seat?). This knowledge (sometimes provided by vibration magnitudes declared by the supplier of the machine or tool) may assist the selection of control measures but will not usually result in a conclusion that there is zero risk.

The control measures might include limitations to the duration of exposure to vibration (e.g., limiting tool use to durations that will not exceed an exposure action value). Although a potentially useful measure this cannot be assumed to eliminate risk, because of the large uncertainties in the prediction of risk. A quantitative control measure should therefore be accompanied by qualitative control measures including checking the implementation of any limit to exposure duration, as well as warning, training, and some form of health surveillance.

\subsection{Flag weaving and flag waving}

Scientists seek to build understanding and may claim to be trying to 'weave' the best flag for 'waving'. Their contributions to the dose-response relationships may improve the prediction of risk, but flag waving cannot wait for scientists to weave this complex story. Furthermore, although improved understanding may lead to a better flag, this understanding will not be sufficient to decide when the flag should be waved. As shown in Figure 2, flag waving can be influenced by, but not solely dependent on, an understanding of the dose-response relationships that guide the definition of measures of exposure severity. Flag weaving and flag waving are different tasks with different objectives. There is a role for those best able to contribute to the making of flags and also a role for those best able to decide whether a flag should be waved.

Confusion between flag weaving and flag waving can impede both the weavers and the wavers. With the promulgation of standardised methods for measuring, evaluating, and assessing exposures it can be assumed that flag waving has become possible because expert weaving has provided a sufficiently 'complete' understanding for all the risks to be identified and controlled. This illusion wrongly suggests there is little 
practical need for better understanding: no need for further weaving to guide the wavers and others in understanding how to predict and control the risks from exposures to vibration and mechanical shock!

\section{Conclusions}

The evaluation of the severity of an exposure to vibration and mechanical shock can inform a risk assessment and can be sufficient to identify a risk. However, the severity of exposure to vibration or shock is often not sufficient to identify risk and never sufficient to control a risk.

Uncertainties associated with measuring exposures to vibration and shock and quantifying their severity, and uncertainty as to the mechanisms involved in injury caused by vibration and shock, mean that factors other than a standardised measure of vibration severity should be considered when deciding whether measures are needed to control the risks. Some of the factors to consider when controlling risks associated with exposure to vibration and shock are identified in Figure 2.

Methods of evaluating vibration severity should be judged on how they encourage change that helps to control risk and not only on their use to assess vibration severity. Accurate quantification of the severity of exposures to vibration or shock is not needed to identify a risk, but may be helpful when modifying an exposure to control a risk.

\section{References}

Griffin, M. J. 2004. "Minimum health and safety requirements for workers exposed to hand-transmitted vibration and whole-body vibration in the European Union; a review". Occupational and Environmental Medicine, 61, 387-397.

Griffin, M. J., M. Bovenzi, and C. M. Nelson. 2003. "Dose response patterns for vibration-induced white finger". Occupational and Environmental Medicine, 60, 16-26.

Bovenzi, M. 2009. "Metrics of whole-body vibration and exposure-response relationships for low back pain in professional drivers: a prospective cohort study". International Archives of Occupational and Environmental Health, 82:893-917. 
Table 1 Logical process for developing and promulgating dose-response information.

(i) Define the type, severity, and probability of the disorder of interest;

(ii) Identify the variables to be included in the measure of dose (e.g., magnitude, frequency, direction and duration);

(iii) For each variable in the measure of dose, discover and quantify causal associations with the chosen measure of disorder, and consider interactions between variables;

(iv) Quantify the uncertainty in the dose-response associations;

(v) Identify the ranges of conditions (e.g., frequencies, directions, and durations) where no association has been established;

(vi) Identify other factors that are not included in the quantified association but may influence the dose-response relationship;

(vii) Estimate the uncertainty associated with the other factors;

(viii) Make it clear when promulgating a dose-response relation what is known and what is assumed. 


\section{Figure captions}

Figure 1 Variables in a dose-response relationship for hand-transmitted vibration or whole-body vibration and mechanical shock.

Figure 2 Process for identifying and controlling risks from human exposure to vibration or mechanical shock. The raised flag indicates that one or more control measure is required. A 'susceptible person' is someone at greater risk than those normally exposed to a similar motion (e.g., extreme age or pregnant), and an 'unusual situation' is one that differs from the norm for the type of vibration or shock (e.g., unusual posture, extreme exposure duration). 


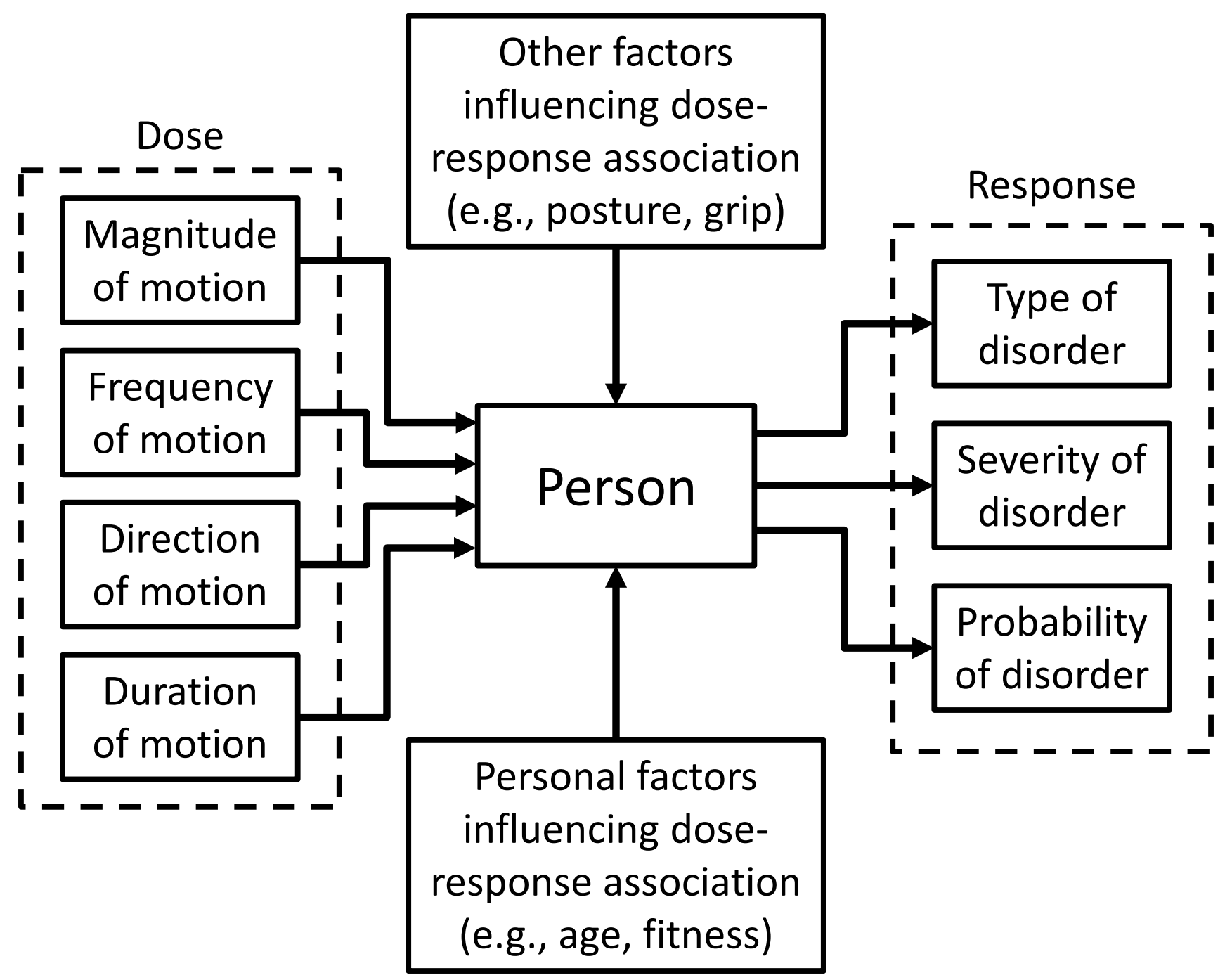


Susceptible person

Exposure severity

Evidence of risk

Unusual situation

Control measures required: raise flag!

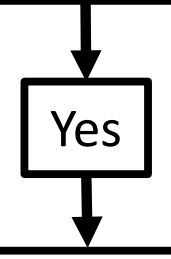

Any foreseeable risks associated with exposure to vibration or mechanical shock?
Type of job associated with risk

Type of machine, tool, or vehicle associated with risk

Environment associated with risk

\section{Consider:}

\section{Available Control Measures}

(i) Elimination at source

(ii) Reduction at source

(iii) Reduced transmission

(iv) Regular warning

(v) Regular training

(vi) Select tools and machines

(vii) Maintain machines and environment

(viii) Control exposure durations

(ix) Optimise posture, clothing, etc.

(x) Health surveillance

(xi) Measure and evaluate exposures if finding will affect control measures.

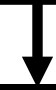

Implement control measures

Regular review 